\title{
Predicting endemic cholera: the role of climate variability and disease dynamics
}

\author{
M. Pascuall ${ }^{1, *}$, L. F. Chaves ${ }^{1}$, B. Cash $^{2}$, X. Rodó ${ }^{3}$, Md. Yunus ${ }^{4}$ \\ ${ }^{1}$ Department of Ecology and Evolutionary Biology, University of Michigan, Ann Arbor, Michigan 48105, USA \\ ${ }^{2}$ Center for Ocean-Land-Atmosphere Studies, Calverton, Maryland 20705-3106, USA \\ ${ }^{3}$ ICREA, Climate Research laboratory, Barcelona, Catalunya, Spain \\ ${ }^{4}$ International Center for Diarrheal Disease Research, Dhaka 1000, Bangladesh
}

\begin{abstract}
Retrospective studies of cholera time series in Bangladesh have established a role of the El Niño Southern Oscillation (ENSO), but also of the non-linear dynamics of the disease itself, through changes in the population levels of immunity in this endemic region. The prediction ability of a semi-mechanistic time series model that incorporates both these elements is examined. Results show that ENSO is a key covariate and confirm the importance of its interplay with immunity levels, now from the perspective of prediction. They support the feasibility of using the model as a forecasting tool: the lack of extreme events between 2001 and 2005 would have been anticipated with $75 \%$ confidence half a year ahead with a model fitted to data up to 2000. Long-term change in the transmission rate, the non-mechanistic part of the model, sets limits to the forecasting horizon because of a breakdown in its relationship with river discharge towards the end of the 1990s. We discuss this and other limitations of the approach as well as future directions related to the development of an early warning system for cholera in this region.
\end{abstract}

KEY WORDS: Cholera prediction $\cdot$ ENSO $\cdot$ Climate forcing $\cdot$ Population immunity $\cdot$ Early-warning systems $\cdot$ Time series model $\cdot$ TSIRS $\cdot$ Nonlinear disease dynamics

\section{INTRODUCTION}

Cholera outbreaks in endemic regions exhibit both seasonality and interannual variability, with significant differences in size from year to year. There has been a long history of trying to explain these interannual cycles, the period of which varies from 3 to $8 \mathrm{yr}$, based on associations with climate (e.g. Russell 1925). The reemergence of cholera in Peru in 1991 to 1992, after the absence of the disease in South America for almost a century, which coincided with an El Niño event, motivated the hypothesis that the El Niño Southern Oscillation (ENSO) is a driver of cholera dynamics (Epstein et al. 1993, Colwell 1996). This hypothesis was tested quantitatively in endemic regions where the disease has been present for a long time and surveillance disease data encompass multiple climate events (Pascual et al. 2000, Bouma \& Pascual 2001, Rodó et al. 2002, Koelle et al. 2005). It was also addressed at global scales by considering the emergence of the disease on different continents over the last century (IPCC 2007). Thus, quantitative evidence has accumulated for a role of ENSO in cholera dynamics in Bangladesh, with an increase in both transmission rates and cases following the warming of the Pacific at lags from 9 to 12 mo. These studies have also illustrated the complexity of the interaction between climate variability and non-linear disease dynamics. In particular, the size of outbreaks is also influenced by the fraction of susceptible individuals in the population, the temporal variability of which is, in turn, determined by the processes of transmission and immunity (Koelle \& Pascual 2004, Koelle et al. 2005).

Given recent advances in the time series modeling of cholera, it is timely to ask whether existing models provide a useful tool for forecasting outbreaks. The interest in the predictive ability of statistical or mathematical models of disease is evident in the literature on the development of climate-based, early warning systems for infectious diseases that are environmentally driven (e.g. Gill 1938, Connor et al. 1999, Hay et al. 2003). 
Efforts in this direction are underway, for example, for epidemic malaria in transition regions, as illustrated by the work in Botswana based on rainfall variability and correlative approaches (Thomson et al. 2005, 2006). We are not aware of any similar efforts with dynamical models that explicitly incorporate immunity (but for a time series approach motivated by these models, see Abeku et al. 2004). Furthermore, one challenging test that is seldom considered is the ability of quantitative approaches to forecast 'out-of-fit' data, that is, data not previously used to fit the model itself. A recent exception is found for American cutaneous leishmaniasis, for which a time series model that incorporates ENSO was better able to forecast cases $1 \mathrm{yr}$ ahead than a null model without any climate information, or equivalent formulations with only regional climate variables (Chaves \& Pascual 2006).

In the present study, we revisit semi-mechanistic model of cholera previously developed and parameterized for a time series of monthly cases in Matlab, Bangladesh, for the period from 1966 to 2002. We examine its ability to both predict outbreaks in the past (1985 to 2000) and to forecast the future (2000 to 2005). Hindcast prediction addresses the loss of predictive ability resulting from simulating the non-linear model forward in time, while forecasting directly addresses the performance of the model for out-of-fit data. Results confirm the importance of the interplay between the susceptible fraction in the population and climate variability (ENSO),
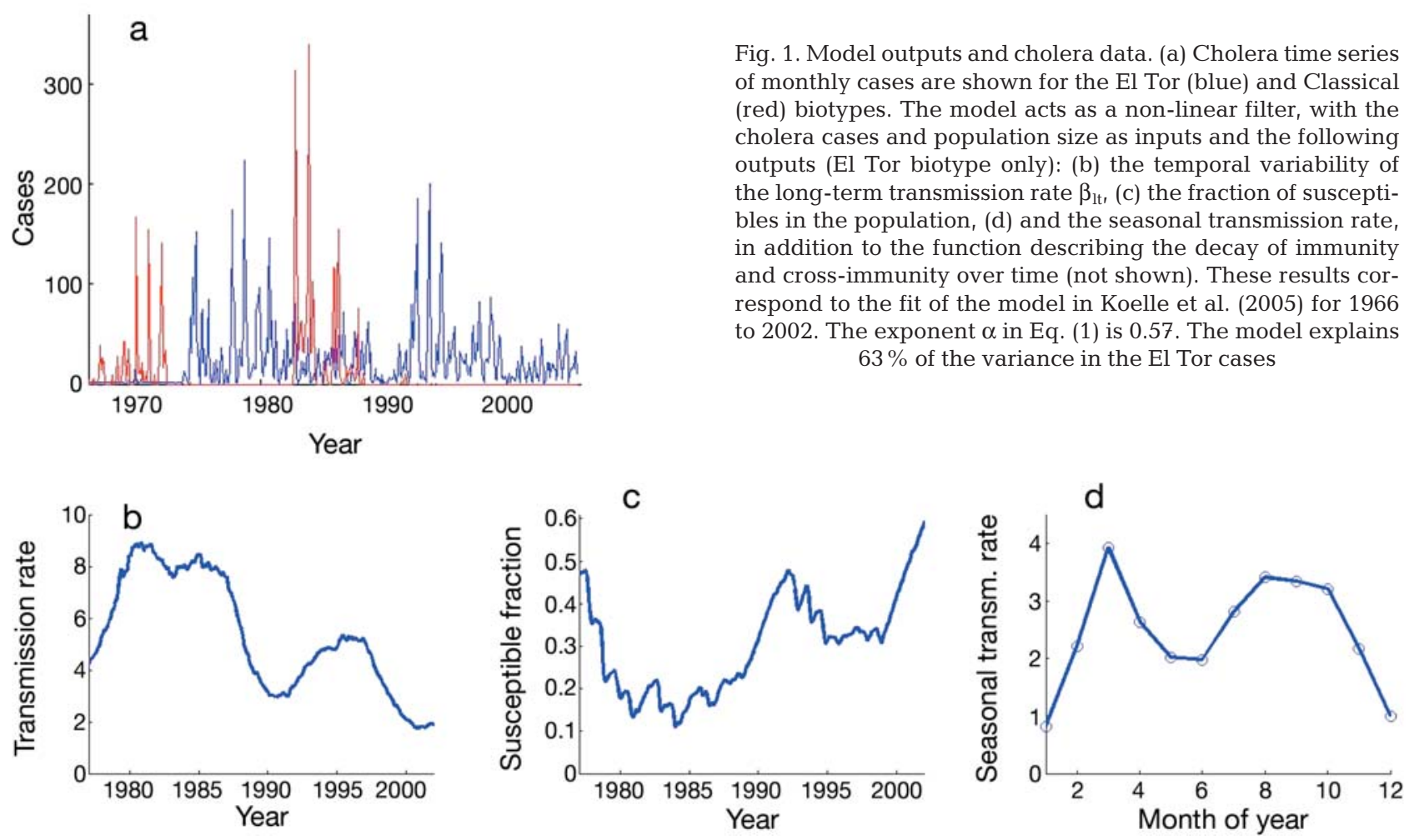

Fig. 1. Model outputs and cholera data. (a) Cholera time series of monthly cases are shown for the El Tor (blue) and Classical (red) biotypes. The model acts as a non-linear filter, with the cholera cases and population size as inputs and the following outputs (El Tor biotype only): (b) the temporal variability of the long-term transmission rate $\beta_{\mathrm{lt}}$ (c) the fraction of susceptibles in the population, (d) and the seasonal transmission rate, in addition to the function describing the decay of immunity and cross-immunity over time (not shown). These results correspond to the fit of the model in Koelle et al. (2005) for 1966 to 2002. The exponent $\alpha$ in Eq. (1) is 0.57 . The model explains

but now from the perspective of prediction. They also support the feasibility of using the model as a forecasting would have been correctly anticipated half a year ahead with $75 \%$ confidence. Finally, ENSO appears to signifithe lis ments for its improvement.

\section{DATA}

The cholera data are monthly cases for the 2 biotypes of Vibrio cholerae known as El Tor and Classical tional Center for Diarrheal Disease Research (ICDDR, B) in Matlab, Bangladesh, from 1966 to 2005. These 2 terium within the pathogenic Serogroup 01. Our model focuses on the dynamics of El Tor, which has replaced Classical over time and whose dynamics continue to be Classical are only intermittent in the series, with the last epidemics observed in the 1980s (Fig. 1a). The new ered here because it has no cross-immunity with El Tor and therefore no mechanistic link to its dynamics. It has also failed to displace El Tor and has caused a negligible number of cases in Matlab in recent years.

\section{$63 \%$ of the variance in the El Tor cases}


For ENSO, the index known as Niño3.4 is used here (obtained from www.cpc.ncep.noaa.gov/data/indices/ sstoi.indices).

We also consider a regional environmental variable, the monthly Brahmaputra river discharge from 1977 to 2005 (gauge data in $\mathrm{m}^{3} \mathrm{~s}^{-1}$ at the first station in Bangladesh). Previous analyses have shown that the lowfrequency variation (periods of $>7 \mathrm{yr}$ ) in the anomalies of this variable is negatively correlated with the longterm transmission rate of cholera $(\mathrm{r}=-0.9278, \mathrm{p}<0.02$, lag $=7 \mathrm{mo}$, significance determined with a bootstrap method that generates surrogate time series for one of the variables by randomizing the phases but preserving the power spectrum and autocorrelation function; Koelle et al. 2005).

\section{METHODS}

We summarize here the main features of the model (for details see Koelle \& Pascual 2004, Koelle et al. 2005). Epidemiological models for the population dynamics of infectious diseases typically divide the population into a number of classes or compartments for the susceptible, infectious, and recovered individuals and follow changes in these variables in continuous time (Anderson \& May 1991). When recovered individuals have temporary immunity and return to the susceptible class after losing immunity, the models are known as SIRS. Discrete formulations of these types of models have been formulated to analyze epidemiological time series and fit their parameters; they are known as TSIR or TSIRS for permanent and temporary immunity, respectively (e.g. Finkenstadt \& Grenfell 2000, Koelle \& Pascual 2004). The discrete formulation implicitly assumes that the time step coincides with the generation time of the infectious class. In the TSIRS for cholera, 2 equations describe the dynamics of the disease. The first one is a non-linear transmission equation of the form:

$$
I_{t+1}=\beta_{t} I_{t}^{\alpha}\left(\frac{S_{t}}{N_{t}}\right) \varepsilon_{t}
$$

where $I_{t}$ is the number of individuals infected (for our model, by the biotype El Tor), $S_{t}$ is the number of individuals susceptible to El Tor, $N_{t}$ is the total population size, and $\varepsilon_{t}$ is a multiplicative noise term, all at time $t$. The exponent $\alpha$ is used to incorporate deviations from the random mixing assumption (e.g. Roy \& Pascual 2006). The pathogen transmission rate $\beta_{t}$ is a key parameter which we specifically let vary in time, to represent the effect of extrinsic drivers on the transmission rate. This parameter corresponds to the number of contacts per infectious individual per unit time, multiplied by the probability that a contact with an infectious individual leads to infection. For cholera, contacts reflect fecal-oral transmission via food and water and/ or environmental transmission via contamination of aquatic environments used by humans. $\beta_{t}$ contains both a seasonal component $\beta_{\text {seas }}$ and a longer-term component $\beta_{\mathrm{lt}}$, such that $\beta_{t}=\beta_{\text {seas }} \beta_{\mathrm{lt}}$ in transmission rates. In particular, this formulation allows for mechanisms that influence interannual variability in $\beta_{t}$ as the result of a modulation of the seasonality. A second equation specifies the number of susceptible individuals at time $t$ :

$$
S_{t}=N_{t}-\sum_{i=0}^{m}\left(\kappa_{i} I_{t-i}\right)-\sum_{i=0}^{m}\left(\kappa_{i}^{C L} I_{t-i}^{C L}\right)
$$

where $N_{t}$ is the current population size at time $t$, $\sum_{i=0}^{m}\left(\kappa_{i} I_{t-i}\right)$ is the total number of individuals recovered from an El Tor infection and immune to El Tor reinfection, and $\sum_{i=0}^{m}\left(\kappa_{i}^{C L} I_{t-i}^{C L}\right)$ is the total number of individuals recovered from infection by a second biotype known as Classical and immune to El Tor reinfection. The functions $\kappa_{i}$ and $\kappa_{i}^{C L}$ describe the decay of immunity and cross-immunity, respectively, with the subscript $i$ indicating the number of months since infection and the values of $\kappa$ and $\kappa_{i}^{C L}$ being the degrees of immunity an individual has $i$ months after being infected. The model focuses on the dynamics of El Tor because this strain has displaced Classical in the past 2 decades and infections display a continuous temporal record (Fig. 1a). The cases for the other strain, Classical, display intermittent behavior and disappear for those last decades; they are used as an input to model the dynamics of El Tor given the existence of cross-immunity. Eqs. (1) and (2) are combined into a single expression relating incidence levels in the present to those in the past.

The model is semi-parametric or semi-mechanistic because it combines an explicit formulation of transmission with the completely unspecified variation of a key parameter, the (long-term) transmission rate. Thus, the model acts as a non-linear filter, with the time series of cases and population sizes as inputs and several outputs, including the decay of immunity, the reconstruction of susceptibles over time (Fig. 1), the seasonal transmission rate (Fig. 1), and the (long-term) temporal variability of the transmission rate (Fig. 1).

There are 2 places in the model where climate forcing can play a role: the variation of the long-term transmission rate $\beta_{1 t}$ and the residuals $\varepsilon_{t}$. After the model is fitted, these patterns of variation can be compared to climate variables for evidence of forcing. While this comparison was carried out in Koelle et al. (2005) by adding these terms and decomposing the resulting signal into fast and slow components (with periods smaller and larger than $7 \mathrm{yr}$, respectively), we take here a more direct approach and analyze the (linear) 
correlation between specific months of the residuals $\varepsilon_{t}$ and an index of ENSO (Niño3.4). Specifically,

$$
\varepsilon_{\mathrm{mo}}=a \times \mathrm{Niño} 34_{\mathrm{Jan}}+b+e(t)
$$

where the $\varepsilon_{\mathrm{mo}}$ denotes the residuals $\varepsilon_{t}$ for a given month, say September, Niño34 Jan denotes the ENSO index in January, $e(t)$ corresponds to the errors of this linear regression, that is, to the variability in $\varepsilon_{t}$ that remains unexplained by ENSO, and $a$ and $b$ to its slope and intercept. Three specific months show significant associations: July, August, and September. We use these associations as the basis to incorporate this climate covariate into the model. Thus, the model is composed of Eqs. (1) and (2). When we further use Eq. (3) to specify $\varepsilon_{t}$ for specific months as a function of the January Niño3.4 in the transmission Eq. (1), we refer to this system as the model with ENSO.

We evaluate 2 aspects of the predictive ability of the model. The first one concerns the loss of predictive ability as the model is iterated forward in time. For this, we consider the El Tor monthly cases for the period between 1985 and 2000, which encompass 3 main El Niño events: 1986/1987, 1991/1992, and 1997/1998. For each time $t$ (i.e. each month), we simulate the model 7 mo ahead and compare this 7 mo lead prediction with the corresponding observation. A measure of predictive ability is given by the so-called prediction $\mathrm{r}^{2}$, which normalizes the error of the predictions using the variance of the time series and substracts this normalized error from 1 . The closer this value is to 1 , the higher the model's predictive ability. Values close to zero or negative indicate poor performance.

To provide a measure of the uncertainty in the predictions, the simulations are stochastic and incorporate the multiplicative noise specified by the residuals $\varepsilon_{t}$ by randomly sampling these terms from the values for the corresponding month. In the model with ENSO, this random sampling applies only to the months for which there is no effect of this covariate. For the other months, August, September, and October, the noise term contains 2 different components: the value predicted by the regression with January sea surface temperatures (SST), and a random sampling from the residuals of this regression, that is, from e(t) in Eq. (3) (see Fig. 2) From 500 simulations, we obtain the median and the 25 and $75 \%$ percentiles for the distribution of predictions at each time. A prediction horizon of 7 mo is selected because the shorter lag with January SSTs, that for cholera in August, is of $7 \mathrm{mo}$; therefore, longer simulations would require a prediction of the covariate itself. An alternative scheme would be to predict February, March, April, and so on, until December from every January, with a varying prediction horizon depending on the month. Similar results to those presented here for 7 mo were obtained, and are, therefore, not shown.
In this first step, we take the values of $\beta_{\text {lt }}$ as given. However, because this parameter constitutes the nonparametric part of the model, its temporally varying values would be unknown as the model is simulated forward. We therefore explore different treatments of $\beta_{l t}$, including setting it to a constant value for the whole prediction interval of the simulation and modeling its variation as a function of a regional climate variable, the Brahmaputra river discharge anomalies (Table 1). River discharge was obtained by averaging daily values per month, ignoring missing values. The monthly
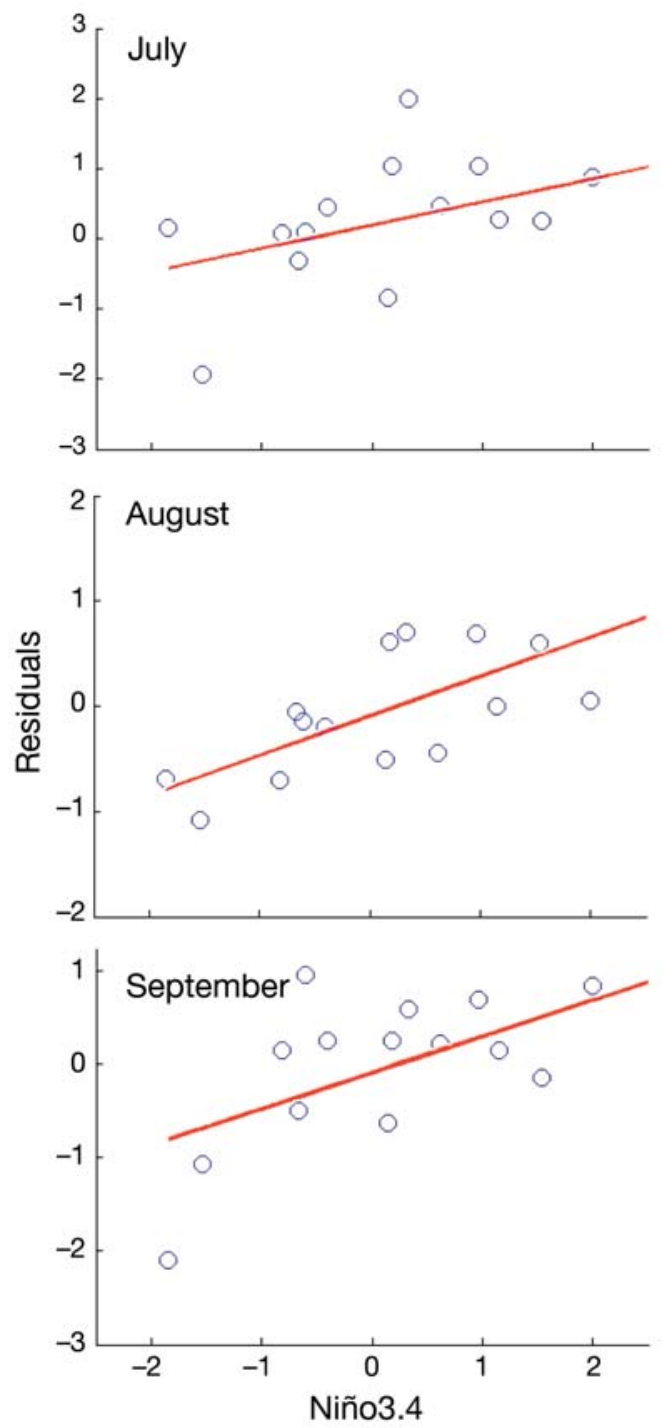

Fig. 2. Model residuals $\varepsilon_{t}$ (Eq. 1) for the months of July, August, and September as a function of the El Niño Southern Oscillation (ENSO) index known as Niño3.4 in January of the same year. The red lines show the fit of a linear regression to these data (Eq. 3). Significant positive correlations are found between January warming in the Pacific and the residuals of these months (September: $\mathrm{r}=0.61, \mathrm{p}=0.01, \mathrm{a}=0.39, b=-0.09$; August: $\mathrm{r}=0.75, \mathrm{p}=0.001, a=0.37, b=-0.09$; July: $\mathrm{r}=0.47$, $\mathrm{p}=0.08, a=0.33, b=0.19$ ) 
Table 1. Parameters for the linear regression of the longterm transmission rate $\beta_{\mathrm{lt}}(1977$ to 1998) as a function of river discharge (Eq. 4). ${ }^{* * *} \mathrm{p}<0.001$

\begin{tabular}{|lcrc|}
\hline Estimated & SE & \multicolumn{1}{c|}{$t$} & $\operatorname{Pr}(>|t|)$ \\
\hline$\mu=4.952939$ & 0.087429 & 56.651 & $<2 \times 10^{-16 * * *}$ \\
$\alpha_{1}=-0.236410$ & 0.023314 & -10.140 & $<2 \times 10^{-16 * * *}$ \\
$\alpha_{2}=0.617220$ & 0.062062 & 9.945 & $<2 \times 10^{-16 * * *}$ \\
$\alpha_{3}=-0.453610$ & 0.046532 & -9.748 & $<2 \times 10^{-16 * * *}$ \\
$\alpha_{4}=0.072646$ & 0.007784 & 9.332 & $<2 \times 10^{-16 * * *}$ \\
\hline
\end{tabular}

data was then filtered using a low pass filter (Cazelles et al. 2007) to separate low-frequency (periods $>7 \mathrm{yr}$ ) from high-frequency (periods $<7 \mathrm{yr}$ ) components in the series following Koelle et al. (2005). This time scale was explicitly chosen to separate variability that may be driven by ENSO from that whose drivers are not related to ENSO (periods $<7$ and $>7 \mathrm{yr}$, respectively). With this filtered signal the following linear model was fitted to $\beta_{1 \mathrm{t}}$ :

$$
\begin{aligned}
\beta_{\mathrm{lt}}(t)= & \mu+\alpha_{1} \operatorname{RD}(t-4)+\alpha_{2} \mathrm{RD}(t-6)+ \\
& \alpha_{3} \mathrm{RD}(t-7)+\alpha_{4} \operatorname{RD}(t-8)+\varepsilon(t)
\end{aligned}
$$

where the lags for the covariate, river discharge (RD), were chosen as those that maximize cross-correlations, following Royama (1992). A model selection criterion,
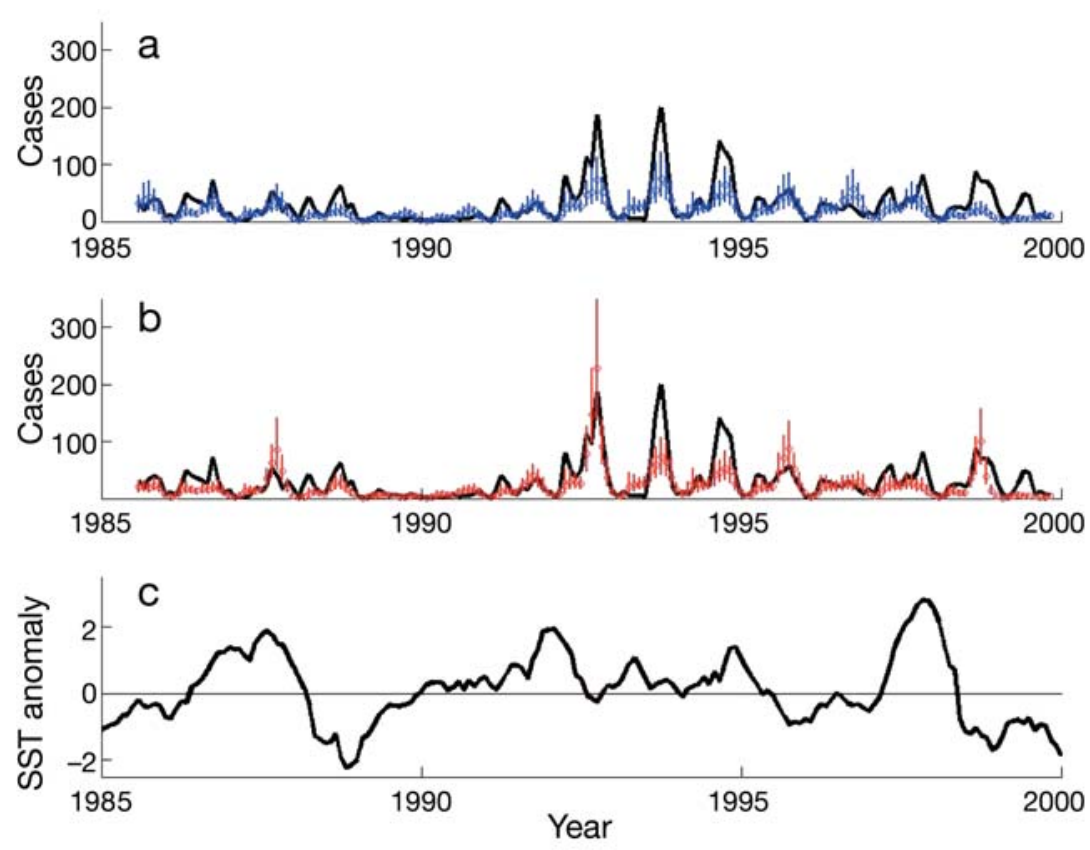

Fig. 3. $(a, b)$ Comparison of 7 mo lead predictions to data (black) for the TSIRS model (a) without and (b) with ENSO as a covariate (blue and red, respectively). The median of 500 stochastic simulations is shown for each time, together with the 25 and $75 \%$ percentiles. (c) Sea surface temperature (SST) anomalies corresponding to the ENSO index Niño3.4. The model with ENSO (b) more accurately predicts the different size of the outbreaks that follow the El Niño events of 1986/1987, 1991/1992, and 1997/1998. Note that similar values of SST anomalies can result in considerable differences in the size of predicted epidemics (compare 1987 to 1992). The predictive $\mathrm{r}^{2}$ of the model that incorporates ENSO equals $78 \%$ (vs. $71 \%$ when this covariate is not taken into account) the adjusted $\mathrm{r}^{2}$ (Faraway 2004), shows that consideraof the ENSO index is not needed, as expected.

hecond aspect considers forecasting accuracy for 2001 to 2005 to evaluate predictions. These forecasts are again generated by stochastic simulations of the the previous values needed to compute the susceptible fraction at time $t$ ), then moving this initial observation forward by 1 mo and repeating the 7 mo simulation, model for each additional observation, and obtaining this parameter up to time $t$. This value is then kept constant for the $7 \mathrm{mo}$ of the simulation. To evaluate the forecasts, we use, in addition to the prediction $\mathrm{r}^{2}$, a less stringent but more practical criterion that considers the ability of the model to anticipate extreme events. We define an extreme outbreak as one that surpasses the $90 \%$ percentile of the distribution of cases (obtained from the 1966 to 2000 time series). We consider the median of 500 simulations and determine the highest percentile of the distribution of predictions for which we do not cross this threshold, to establish a measure of the confidence with which we can say that an extreme event will not occur.

\section{RESULTS}

The residuals of the model exhibit significant correlations with SST anomalies in the Pacific (Niño3.4) for 3 specific months only: July, August, and September (Fig. 2). Warming in January is followed by an increase in the residuals of these 3 mo preceding or coinciding with the fall peak, typically in September for the El Tor biotype. A linear regression is fitted to these residuals as a function of Niño3.4 as a simple way to incorporate the effect of ENSO to the dynamics.

Fig. 3a,b shows the 7 mo lead predictions for the period from 1985 to 2000, obtained by taking each observation (and preceding cases) as initial conditions and simulating the model for 7 consecutive months stochastically with 
and without ENSO, respectively. The model that incorporates ENSO is better able to predict the different sizes of the outbreaks that follow the 3 main El Niño events in 1986/1987, 1991/1992, and 1997/1998, although the 2 other outbreaks that accompany the sustained warming conditions in 1992 to 1995 are not captured. It is interesting to compare the response of the model for the first 2 events, when SST anomalies are of very similar magnitude, but the resulting cholera outbreak is much larger in 1992 than in 1987 (Fig. 3). Note that this is not the result of a higher value of the transmission rate $\beta_{1 t}$ (Fig. 1b). In fact, this parameter is higher in 1987. Thus, this difference in the disease response must follow from the larger number of susceptibles in 1992. The previous epidemics of the 1970s and early 1980s have depleted the pool of susceptibles, and the dynamics of cases can only weakly respond to warming in 1987 (Fig. 1a). Notice that periods of high outbreaks are followed by low outbreaks in an alternating pattern lasting almost a decade. This reflects the buildup and waning of immunity over several years, with the corresponding periods of high and low response to climate forcing as described in Koelle et al. (2005). In 1987, the disease model is in a refractory state responding weakly to exogenous forcing by ENSO. Differences in the susceptible pool cannot fully explain, however, the size of epidemics when comparing 1992 to 1998 . Here, the decrease in the transmission rate $\beta_{1 \mathrm{t}}$ also plays a critical role.

This brings us to one disadvantage of using a semiparametric model for predictive purposes. We have taken the value of the variable transmission rate $\beta_{\mathrm{lt}}$ as given to focus on the mechanistic part of the model and the interaction of climate forcing with the non-linear dynamics of the disease. Because the variability in this parameter would presumably be unknown for the prediction window, one would like, ideally, to also be able to predict this parameter. In Koelle et al. (2005), a negative and highly significant correlation is shown for $\beta_{\mathrm{lt}}$ and the low-frequency variation of 2 regional climatic variables, namely NEIR (North East Indian rainfall) and river discharge for the Brahmaputra. We investigate here the association with river discharge further by comparing the predicted values of a linear regression fitted to $\beta_{1 \mathrm{t}}$ before 1998 (Eq. 4) to the values of this parameter before and after 1998 (Fig. 4). The goodness of fit for the early part of the $\beta_{\mathrm{lt}}$ time series is remarkably high, and simulations that use the value of $\beta_{1 t}$ predicted using river discharge (Eq. 4) give results similar to those obtained by taking $\beta_{\mathrm{lt}}$ as given (not shown). However, the accuracy of the predicted $\beta_{\mathrm{lt}}$ after 1998 is surprisingly low, which precludes using river discharge as a means to obtain this parameter after 1998.

Given this apparent break in the relationship between long-term transmission and river discharge, we

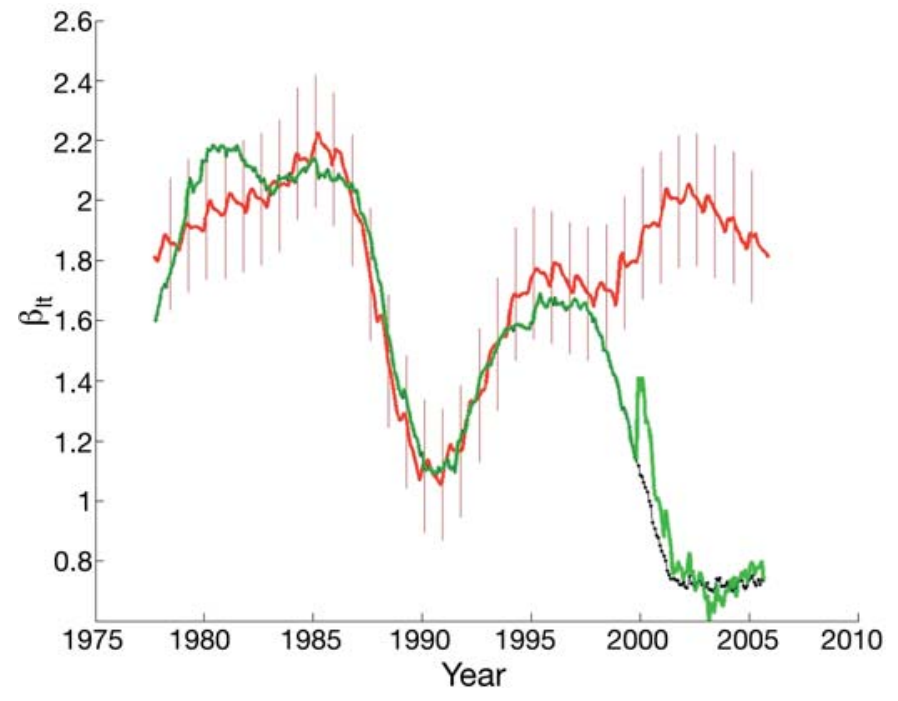

Fig. 4. Long-term transmission rate $\beta_{\mathrm{lt}}$ as a function of river discharge for the model fitted to the whole time series from 1966 to 2005 (black dots, below the green curve before 2000). The plots start in 1977 because the initial part of the data is used as first conditions given the duration of immunity and cross-immunity (Koelle et al. 2005). Values of $\beta_{\text {lt }}$ obtained by fitting the model up to the year 2000, and by successive fits of the model, where 1 data point is added at a time from 2000 to 2005, after that year (green curve). These latter values are used in the forecasts for the period from 2000 to 2005, mimicking the situation of updating the model as new monthly data become available. Predicted values and confidence intervals for a linear regression of $\beta_{\mathrm{lt}}$ as a function of river discharge (Eq. 3) fitted for the data from 1977 to 1998 (red curve). Note that, although these associations provide a reasonable fit, they predict the long-term transmission rate very poorly after 1998

considered that $\beta_{\mathrm{It}}$ remains constant for the prediction interval of $7 \mathrm{mo}$, based on the knowledge that this is a slowly varying parameter (Koelle et al. 2005; for details see also Koelle \& Pascual 2004). This parameter was obtained for each added observation of monthly cases by re-fitting the TSIRS model (Eqs. 1 \& 2). Another parameter that varies in time and is needed for updating susceptibles but is unknown in practice during the prediction interval, is population size. However, this variation has negligible effects, and the long-term trend in population size can easily be projected 7 mo into the future.

Fig. 5 presents the 7 mo lead forecasts for the more recent period from 2001 to 2005, with the model with and without ENSO and a constant $\beta_{1 \mathrm{lt}}$ for each prediction interval. Both models would have predicted the lack of an extreme outbreak for these years with $75 \%$ confidence. That is, the $75 \%$ percentile for the distribution of predicted cases for each month does not cross the threshold that defines an extreme event. Only 1 El Niño event occurred during this period, in 2002. While the fraction of susceptibles in the popula- 

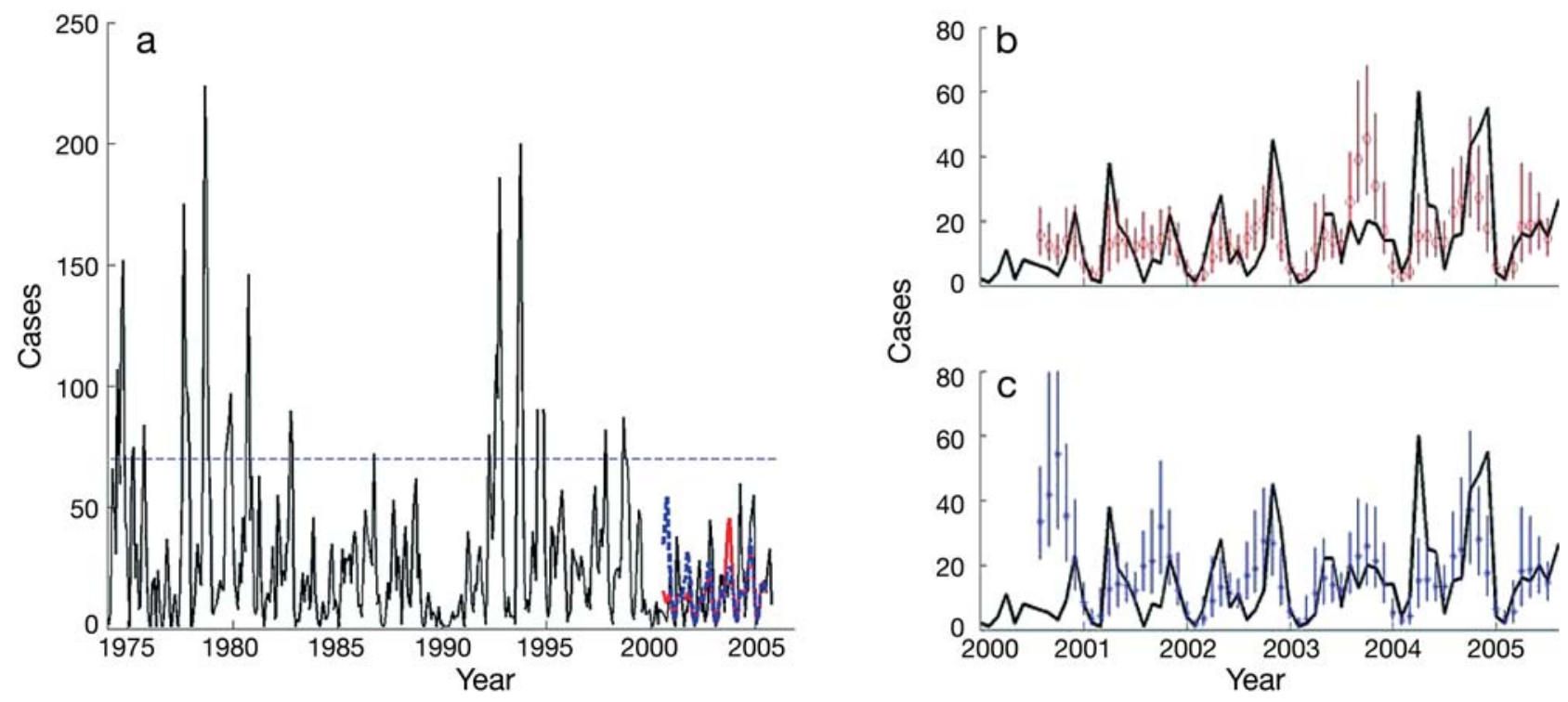

Fig. 5. (a) The 7 mo lead forecasts of out-of-fit data. Cholera cases are shown in black, and the median of 500 forecasts are shown in red and blue for the model with and without ENSO, respectively. The dashed line indicates the $90 \%$ percentile of the distribution of cases, used here to define extreme outbreaks. (b,c) Forecasted years with the 25 and $75 \%$ percentiles of the distribution of predicted values for each month added. The model forecasts the lack of extreme events from 2001 to 2005 with $75 \%$ confidence. The predicted $\mathrm{r}^{2} \approx 98 \%$ is quite high for both models because the variance of the whole time series of cases is used to normalize the errors

tion is high, the long-term transmission rate is low and remains low for this whole period (Fig. 1b,c), precluding, in part, a response to ENSO. Still, the ENSO-based model overestimates the response in 2003. While Bangladesh typically experiences increased rainfall following a winter El Niño event (Cash et al. 2007), there is considerable event-to-event variability. Despite following a warm event, summer rainfall over Bangladesh in 2003 was deficient, and may explain why the model overestimates the cholera response for that year (Fig. 6). In 2001, the model without ENSO overestimates the cases significantly, whereas the model with ENSO accurately predicts the low levels of the disease. This difference is due to the effect of a negative SST anomaly.

\section{DISCUSSION}

This initial exploration of the TSIRS model from the perspective of prediction supports the feasibility of using this

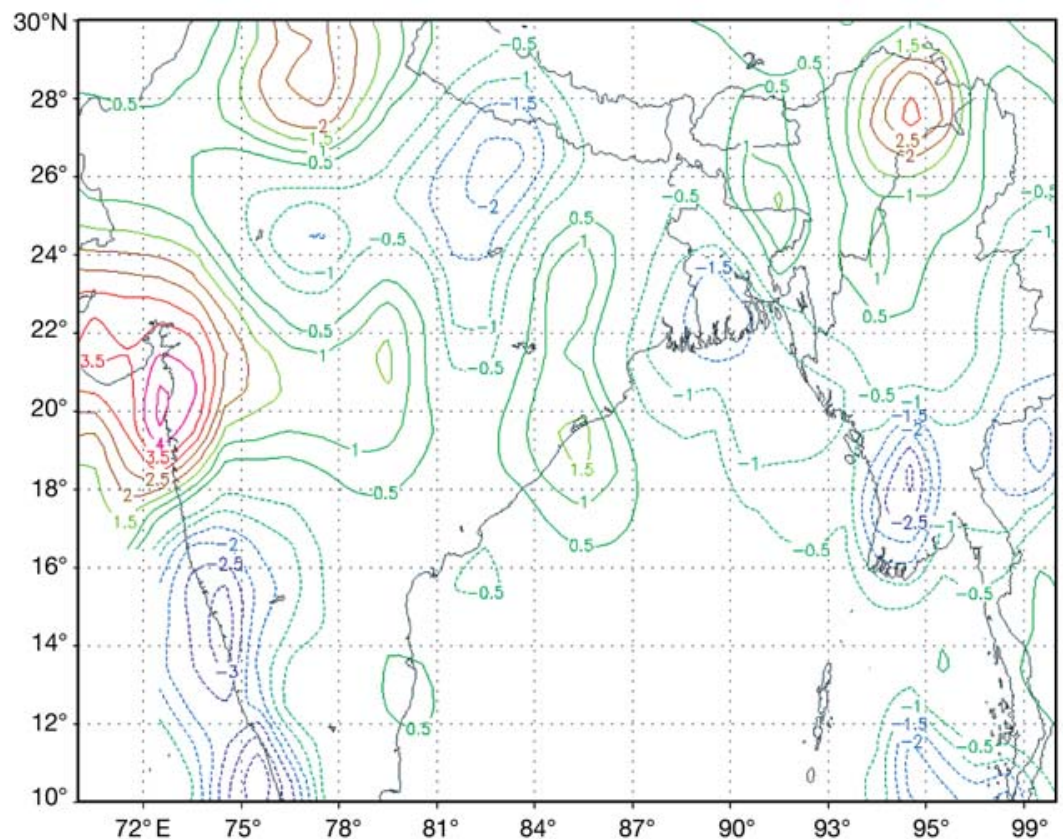

Fig. 6. June-July-August (JJA) rainfall anomaly for the summer of 2003 relative to 1950-2003 climatology, showing a rainfall deficiency over Bangladesh. The pattern is very nearly the opposite of the 1976 to 2002 composite warm-cold anomaly pattern, with dry conditions over south-west India and central-western Bangladesh and wet conditions over north-west and central India, along with wet anomalies surrounding Bangladesh (not shown). The precipitation data are from Chen et al. (2002) 


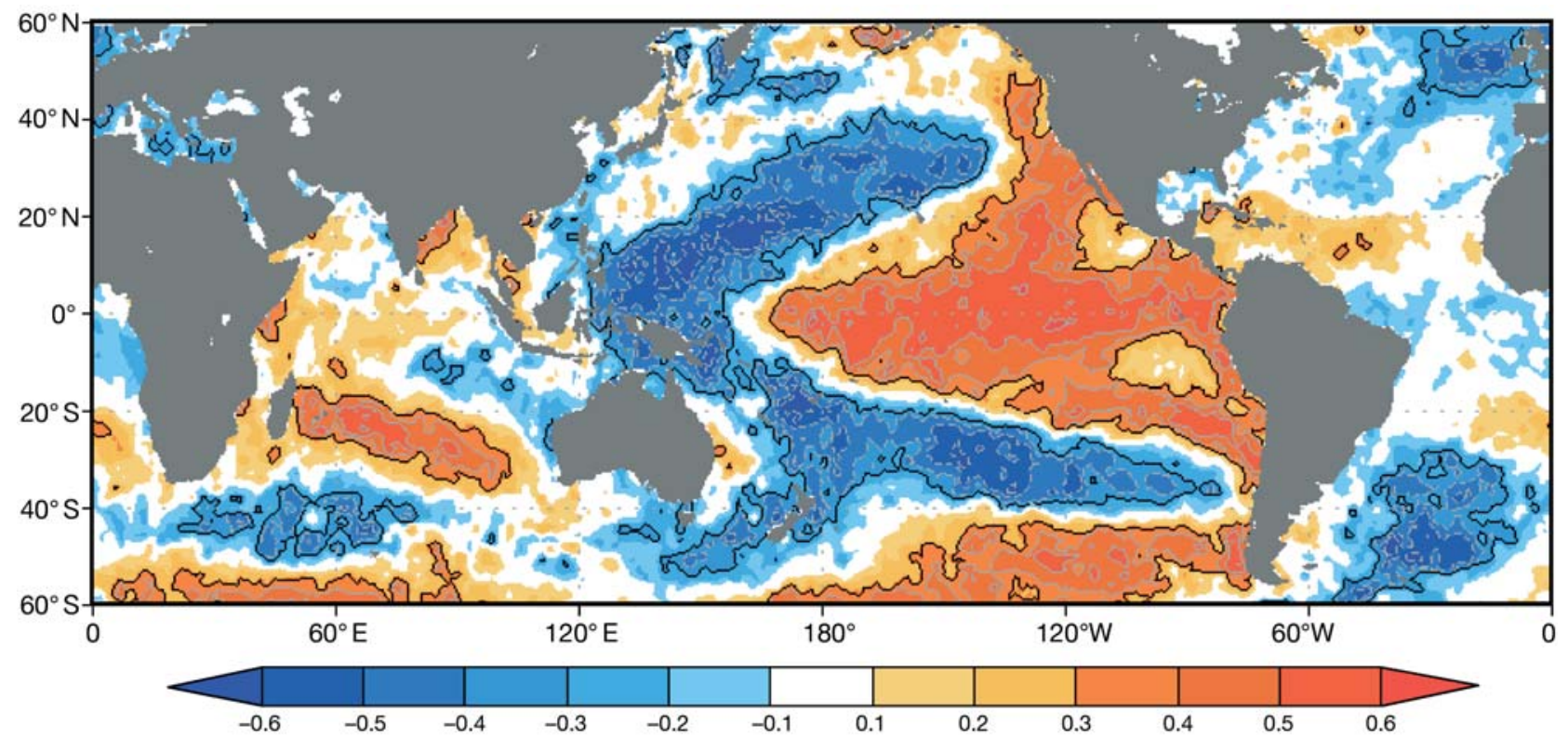

Fig. 7. Rank correlations between September cholera cases and January SST anomalies in a global ocean grid. The black line indicates the $90 \%$ significance level

model for short-term forecasting of cholera in Matlab, Bangladesh. The results for 1985 to 2000 also indicate that ENSO is a useful covariate to include in the model. This variable was incorporated in the model using a simple approach, based on the demonstrated significant and positive association between an index of ENSO, Niño3.4, and the lagged residuals of the transmission equation in the months of July, August, and September. Interestingly, this pattern of association is consistent with that observed for September cases and a global grid of January SST anomalies in the world oceans (Fig. 7). A region of significant rank correlations emerges in the Pacific that corresponds geographically to the pattern of warming observed during El Niño events (Cash et al. 2007).

Results also show that the fraction of susceptibles in the population, and therefore the previous dynamics of the disease, modulate the response to climate and determine the size and accuracy of predicted outbreaks. This is particularly apparent for warming events of similar magnitude that are followed by outbreaks of different sizes, in 1987 and 1992, respectively. While the model for disease dynamics completely fails to predict this difference, the model with SST January anomalies as a covariate succeeds. Not all large outbreaks, however, are successfully predicted by the model. In particular, the 2 epidemics following the one in 1992, during the sustained warming conditions of 1991 to 1995, are missing. Two possible explanations suggest, in turn, future improvements to our approach. First, the particular index of ENSO happens to have lower values than in 1992 for January of those particular years, even if the warming conditions prevail in adjacent months. The use of a single month and a single index may be replaced by a more robust relationship between ENSO and the residuals in the transmission equation based on multiple months and/or multiple indices. In particular, the geographic area of the Pacific used for this index could be replaced by one defined directly by the associations with cholera cases and model residuals (Fig. 7 and Cash et al. 2007). Second, immunity in the model may be too long-lasting, precluding a sequence of extreme outbreaks from occurring. Alternative mechanisms and representations for the difficult problem of immunity in cholera are under investigation (King et al. in press).

The non-parametric component of our model, the long-term variation in the transmission rate, sets a limit to the prediction horizon after 1998 because of the break in the relationship to river discharge. The reason for this break remains an open question: Does it reflect a change in the intrinsic dynamics of cholera or in control measures in this region? Does it reflect a change in the climate measurements themselves? Or does it simply provide an example of correlation not implying causation. Regardless of the answer, we have shown that one feasible approach, in the absence of a good model for the long-term transmission rate, is to assume that this parameter remains constant for the prediction interval, with reasonable results. Alternative models for projecting $\beta_{\text {lt }}$ during the prediction interval should be explored. 
Future improvements of our approach should also examine how to incorporate regional climate variables that mediate the ENSO effect on cholera in Bangladesh. Previous results (Koelle et al. 2005) and on-going studies with coupled ocean-atmosphere climate models (Cash et al. 2007) suggest that regional flooding and precipitation should be considered. Clearly, individual El Niño events can differ in their effects in this region and determine, in part, the particular response of the disease to warming in the Pacific. For example, the June-July-August (JJA) rainfall anomaly for the summer of 2003 (Fig. 6) is very nearly the opposite of the 1976 to 2002 composite warm-cold anomaly pattern (not shown). Thus, the 2002 El Niño is not only weaker, but appears to be followed by different precipitation conditions in Bangladesh than the 1997/1998 event. Under weak El Niños and/or weak coupling between conditions in the Pacific and those in the Indian Ocean, regional drivers may be particularly important.

Another improvement might be found in the statistical model for the residuals as a function of ENSO. While we have used a simple linear regression, it is worth examining whether a non-linear relationship and a differential effect of positive and negative anomalies give better results. Future cholera data may allow us to examine the prediction of extreme events further, since successful forecasting of their occurrence is not necessarily implied by our results on the lack of extreme outbreaks.

Finally, an early warning system should be based on an ensemble of forecasts produced with $>1$ single model, including the full spectrum from non-mechanistic time series methods, such as seasonal autoregressive models (e.g. Chaves \& Pascual 2006) and nonlinear maps based on attractor reconstruction (Dixon et al. 1999, Pascual et al. 2000, Szeliga et al. 2003), to mechanistic representations closer to the mathematical models used in epidemiology with different types of noise (Ionides et al. 2006). Our initial effort indicates that an early warning system is achievable and worth pursuing.

Acknowledgements. The cholera data used for this research study were collected with funding by the International Center for Diarrheal Disease Research (ICDDR,B) and its donors, who provide unrestricted support to the Center for its operations and research. Current donors providing unrestricted support include: the Australian International Development Agency (AusAID), the Government of Bangladesh, the Canadian International Development Agency (CIDA), the Government of Japan, the Government of the Netherlands, the Swedish International Development Cooperative Agency (SIDA), the Swiss Development Cooperation (SDC), and the Department for International Development, UK (DFID). We gratefully acknowledge these donors for their support and commitment to the Center's research efforts. We thank Peter Webster and the Climate Forecast Applications Project at Georgia Tech for the river discharge data, the Fundació 'la Caixa', in particular their Science Museum CosmoCaixa in Barcelona, for the stimulating Symposium on 'New and Re-Emerging Diseases, Populations and Climate', which motivated us to conduct this work, and 3 anonymous reviewers for their useful comments. Support was provided by grants from the National Oceanic and Atmospheric Administration (Oceans and Health Program) and NSF-NIH (Ecology of Infectious Diseases) to M.P.

\section{LITERATURE CITED}

Abeku TA, Vlas SJDE, Boorsboom GJJM, Tagede A and others (2004) Effects of meteorological factors on epidemic malaria in Ethiopia: a statistical modeling approach based on theoretical reasoning. Parasitology 128:585-593

Anderson RM, May RMC (1991) Infectious diseases of humans: dynamics and control. Oxford University Press, Oxford

Bouma MJ, Pascual M (2001) Contributions of climate and geography to seasonal and interannual cycles of endemic cholera in Bengal 1891-1940. In: Special edition on 'Diseases in the Ocean'. Hydrobiologia 460:147-156

Cash BA, Rodó X, Kinter JL III (2007) Links between tropical Pacific SST and the regional climate of Bangladesh: role of the eastern and central tropical Pacific. COLA Technical Report No. 236, Center for Ocean-Land-Atmosphere Studies, Calvertion, MD

Cazelles B, Chavez M, Constantin de Magny G, Géguan J, Hales S (2007) Time-dependent spectral analysis of epidemiological time-series with wavelets. J R Soc Interface. doi:101098/rsif.2007.0212

Chaves LF, Pascual M (2006) Climate cycles and forecasts of cutaneous leishmaniasis, a non-stationary vector borne disease. PLoS Med 3:1320-1328

> Chen M, Xie P, Janowiak JE (2002) Global land precipitation: a 50-yr monthly analysis based on gauge observations. J Hydromet 3:249-266

> Colwell RR (1996) Global climate and infectious disease: the cholera paradigm. Science 274:2025-2031

> Connor SJ, Thomson MC, Molyneux DH (1999) Forecasting and prevention of epidemic malaria: new perspectives on an old problem. Parassitologia 41:439-448

Dixon PA, Milicich MJ, Sugihara G (1999) Episodic fluctuations in larval supply. Science 283:1528-1530

Epstein PR, Ford TE, Colwell RR (1993) Cholera and the environment. Lancet 342:1216-1219

Faraway JJ (2004) Linear models with R. Chapman \& Hall/CRC, Boca Raton, FL

Finkenstadt BF, Grenfell BT (2000) Time series modelling of childhood diseases: a dynamical approach. Appl Stat 49: 187-205

Gill CA (1938) The seasonal periodicity of malaria and the mechanism of the epidemic wave. J. \& A. Churchill, London

Hay SI, Were EC, Renshaw M, Noor AM and others (2003) Forecasting, warning, and detection of malaria epidemics: a case study. Lancet 361:1705-1706

Ionides EL, Breto C, King AA (2006) Inference for nonlinear dynamical systems. Proc Natl Acad Sci USA 103: 18438-18443

IPCC (Intergovernmental Panel on Climate Change) (2007) Climate change 2007: impacts adaptation and vulnerability. Contribution of Working Group II to the Fourth Assessment Report of the Intergovernmental Panel on Climate Change. Cambridge University Press, Cambridge

King AA, Ionides E, Pascual M, Bouma MJ (in press) Inap- 
parent infections and short-term immunity in cholera dynamics. Nature

Koelle K, Pascual M (2004) Disentangling extrinsic from intrinsic factors in disease dynamics: a nonlinear time series approach with an application to cholera. Am Nat 163:901-913

Koelle K, Rodó X, Pascual M, Yunus Md, Mostafa G (2005) Refractory periods to climate forcing in cholera dynamics. Nature 436:696-700

Pascual M, Rodó X, Ellner S, Bouma MJ, Colwell R (2000) Cholera dynamics and El Niño-Southern Oscillation. Science 289:1766-1769

Rodó X, Pascual M, Fuchs G, Faruque S (2002) ENSO and cholera: A nonstationary link related to climate change? Proc Natl Acad Sci USA 99:12901-12906

Roy M, Pascual M (2006) On representing network heterogeneities in the incidence rate of simple epidemic models.

Editorial responsibility: Nils Chr. Stenseth, Oslo, Norway
Ecological Complexity 3:80-96

Royama T (1992) Analytical population dynamics. Kluwer, Amsterdam

Russell AJH (1925) The epidemiology of cholera. Indian J Med Res 13:427-439

Szeliga MI, Verdes PF, Granitto PM, Ceccatto HA (2003) Artificial neural network learning of nonstationary behavior in time series. Int J Neural Syst 13(2):103-109

Thomson MC, Mason SJ, Phindela T, Connor SJ (2005) Use of rainfall and sea surface temperature monitoring for malaria early warning in Botswana. Am J Trop Med Hyg 73:214-221

Thomson MC, Doblas-Reyes FJ, Mason SJ, Hagedorn R and others (2006) Malaria early warnings based on seasonal climate forecasts from multi-model ensembles. Nature 439:576-579

Submitted: April 16, 2007; Accepted: November 17, 2007 Proofs received from author(s): April 19, 2008 